\title{
Forecasting the Price of Gold Using Dynamic Model Averaging
}

\author{
Goodness Aye ${ }^{\mathrm{a}}$, Rangan Gupta ${ }^{\mathrm{a}}$, Shawkat Hammoudeh ${ }^{\mathrm{b}}$, Won Joong Kim ${ }^{\mathrm{c}}$
}

\begin{abstract}
We develop models for examining possible predictors of the return on gold that embrace six global factors (business cycle, nominal, interest rate, commodity, exchange rate and stock price factors) extracted from a recursive principal component analysis (PCA) and two uncertainty indices (the Kansas City Fed's financial stress index and the U.S. Economic uncertainty index). Specifically, by comparing with other alternative models, we show that the dynamic model averaging (DMA) and dynamic model selection (DMS) models outperform not only a linear model (such as random walk) but also the Bayesian model averaging (BMA) model for examining possible predictors of the return of gold. The DMS is the best overall across all forecast horizons. Our result is also robust to a version of the PCA that uses all the 28 potential predictors instead of categorizing them, as well as, alternative out-of-sample period. Generally, all the predictors show strong predictive power at one time or another though at varying magnitudes, while the exchange rate factor and the Kansas City Fed's financial stress index appear to be strong at almost all horizons and sub-periods. However, the forecasting prowess of the exchange rate is supreme.
\end{abstract}

JEL Classification codes: C11, C53, F37, F47, Q02

Keywords: Bayesian, state space models, gold, macroeconomic fundamentals, forecasting

\footnotetext{
${ }^{a}$ Department of Economics, University of Pretoria, Pretoria, South Africa.

Email: rangan.gupta@up.ac.za.

b Lebow College of Business, Drexel University, Philadelphia, United States and IPAG Business School, Paris, France.

Email: hammousm@drexel.edu.

${ }^{\mathrm{c}}$ Corresponding author. Department of Economics, Konkuk University, Seoul, Korea.

Email: wjkim72@konkuk.ac.kr.
} 


\section{Introduction}

Gold serves several functions in the world economy, and its links with financial and macroeconomic variables are quite well-established (Pierdzioch et al., 2014a, b). It has a monetary value and is sought after by central banks to be part of their international reserves which fulfil many purposes (Gupta et al., 2014). ${ }^{1}$ It has industrial uses and can be transformed into jewelry. In modern finance, it is used as a hedge against inflation and a safe haven during crises (Ciner et al., 2013; Ghazali, 2013). Gold has also other distinguished characteristics. Its supply is accumulated over the years and its global annual physical production can be as small as $2 \%$ of total supply, thereby in contrast to other commodities its annual production may not sway its price as other factors do. Moreover, unlike prices of stocks and bonds, the gold price depends on future supply and demand, and thus it is forward-looking.

A myriad of other global factors affect the price of gold (Pierdzioch et al., 2014a), which in turn, can be primarily classified into six categories: The business cycle factor; the nominal factor; the interest rate factor; the commodity factor; the exchange rate market factor; and the stock price factor. As is standard in the literature, one factor is created based on the first principal component of each set or bloc of variables. Thus, for example, for the business cycle factor, we use the first principle component of the four stationary and standardized variables. All the factors are latent, but they still capture the common component of the variables in the group. We add to these six factors, the Kansas City Fed's financial stress index and the U.S. economic policy uncertainty which can impact the gold price returns because gold is used as a safe haven (Baur and Lucey 2010; Baur and McDermott, 2010, 2012; Ciner et al., 2013) and those variables deal with fear and anxiety (Ciner et al., 2013). The business cycle factor (Pierdzioch et al., 2014b) is represented by an economic activity variable, which is the industrial production of the first and second largest economies, namely the United States and China, as well as for Japan and Europe.

\footnotetext{
${ }^{1}$ On an average year, more than $62 \%$ of gold supply is used in jewelry, more than $18 \%$ is used in industrial applications and about $18 \%$ is held in central banks' foreign reserves.
} 
An improvement in this economic activity will increase the industrial and jewelry demands for gold as a result of increased world production and income.

The nominal factor refers to inflation as represented by the CPI for the United States, EU, China and Japan and the money supply growth for the United States and EU. As indicated, gold is a hedge against inflation (Jaffe 1989; Capie et al., 2005; Jensen et al., 2002; Ghazali, 2013).. Moreover, gold prices are sensitive to changes in interest rates which represent the opportunity cost of hoarding gold. This factor includes the interest rates for the U.S. and EU, and LIBOR. Gold is also a commodity that is affected by movement in commodity prices. When there is a commodity boom, gold co-moves in sympathy because there are common macroeconomic variables that comove with related and unreelated commodities (Pindyck and Rotemberg, 1990). The gold price return is also influenced by changes in the foreign exchange markets since gold is considered a currency (Sjaastad, 2008; Sjaastad and Scacciavillani, 1997). This relationship is theoretically explained by the law of one price. A weakness in the dollar exchange rate will motivate investors and traders to seek the safety of gold (Ismail et al., 2009). Finally, gold is an asset class that is related to financial asset classes like the stocks. Gold and stocks may move together but they may depart depending on the business cycle. As a robustness check, conduct recursive PCA on all the 28 variables (which have been previously categorized into the six factors and two individual series as explained above) without categorizing them and subsequently select the optimal number of factors based on Bai and $\mathrm{Ng}$ (2002). The extracted factors are in turn used in forecasting gold returns in a DMA framework.

Against this backdrop, this paper applies the dynamic model averaging (DMA) strategy developed by Raftery et al. (2010), which can also be used for the dynamic model selection (DMS) methodology. In this methodology, a single but potentially different model can be employed as a forecasting model at each point of time to forecast the returns on gold based on the wide array of predictors discussed above, which may move in sync or in different directions. The DMA or DMS seems ideally suited for forecasting a variable as volatile as the returns on 


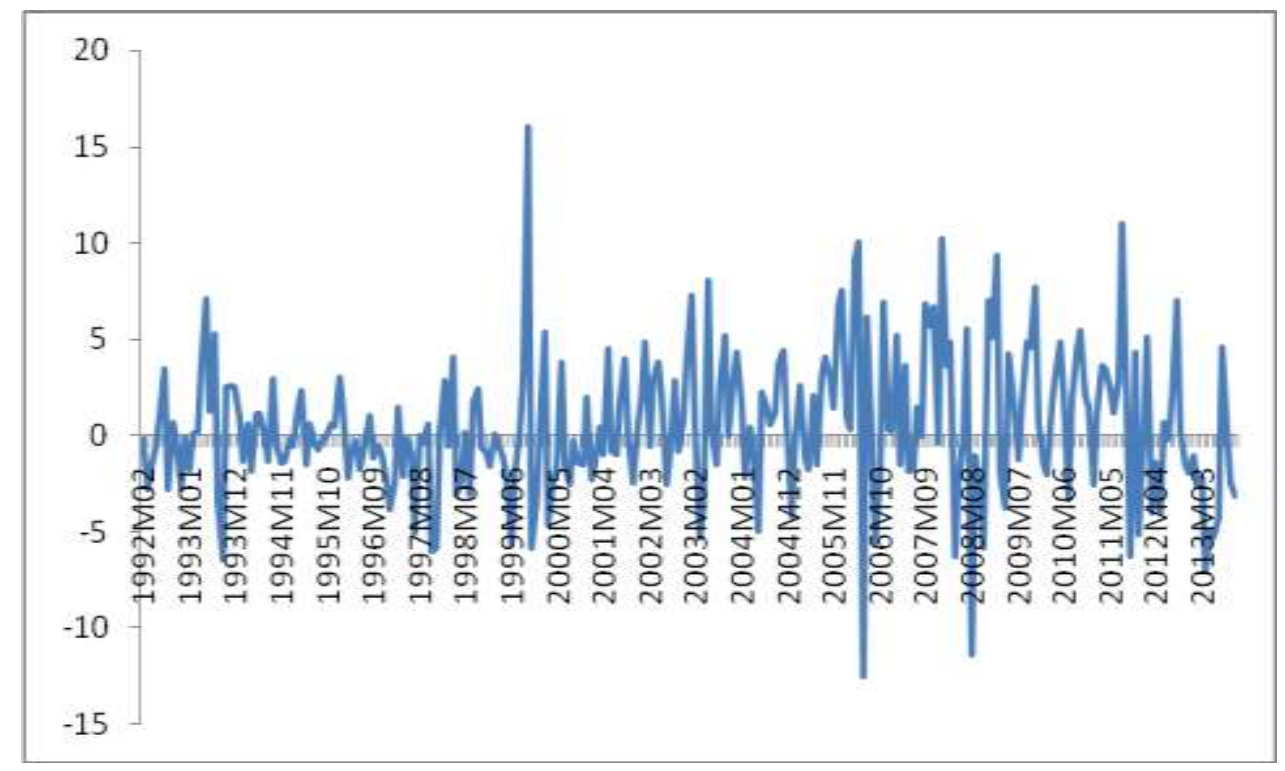

Figure 1: Returns on gold (in percentage, month-on-month)

gold (see Figure 1), which have gone through periods of major events and crises. These methodologies not only allow the forecasting model to change over time, but also the coefficients in each model to evolve over time simultaneously. These two methods involve standard econometric methods used for state space models, such as the Kalman filter, but through some sensible empirical approximations, they achieve vast gains in computational efficiency so as to allow one to apply DMA and DMS despite the computational difficulties. The advantages of using the DMA approach over standard predictive regression methods are three folds. (1) The coefficients on the predictors can change over time, while the conventional use of dummy variables to capture possible structural breaks or recursive methods to take care of time variations is not suited to solve this issue. Hence ideally, one needs to build models designed to capture the changing of slope parameters of the models. (2) Given that we use a wide variety of predictors, the number of potential predictors is in general quite large, thereby in the process making the number of models quite large as well. For instance, if the set of models is defined by whether each of the $m$ potential predictors are included or excluded, then we have $2^{m}$ possible models to work with. This would raise substantial statistical problems involved in model 
selection, which in turn, has resulted in researchers resorting to the Bayesian model averaging (BMA) as in Wright (2008) and Tortora (2010). (3) Finally, the model that is relevant for forecasting can itself potentially change over time due to a change in the set of predictors or with some variables better capable of forecasting during, for example, recessions than expansions. This makes the situation even more complicated, since with $2^{m}$ models the number of combinations of models required to be estimated in order to forecast at time $\tau$ is $2^{m \tau}$ to allow for the possibility of a different model being applicable at each point in time. Even in relatively simple forecasting exercises, it can be computationally impossible to forecast by simply going through all of these $2^{m \tau}$ combinations.

To the best of our knowledge, this is the first study to forecast returns on gold using timevariations in parameters as well as models. Based on monthly data on the predictors and using an out-of-sample period of 1999:10-2013:11 (with an in-sample of 1992:02-1999:09), we compute multi-steps-ahead forecasts for gold returns based on recursive estimation of the models over the out-of-sample period. Note that the start and end-point of our entire sample is purely driven by data availability, while the starting point of out-of-sample corresponds to the period when the returns on gold reached its peak ${ }^{2}$ (see Figure 1).

Among all the models considered, namely the time-varying parameter (TVP), DMA, DMS, Bayesian model averaging (BMA) and the benchmark random-walk (RW) models, the results show that the DMA and DMS models forecast generally well, with DMS being the best overall. These results are supported by two diagnostics, namely the sum of the log predictive likelihoods and the mean squared forecast error (MSFE). It can therefore be concluded that allowing for model uncertainty and not only parameter uncertainty would improve the forecasting performance of these models. One major advantage of the DMA is that the framework is capable of identifying

\footnotetext{
2 The choice of an out-of-sample period is most of the times ad hoc. However, the forecasting literature does tend to choose an out of sample period that is believed to be difficult enough to forecast, say in terms of volatility, at the same time ensuring that the out-of-sample is also large enough. In our case, we chose the starting point of the outof-sample that corresponds to the peak, since it might be difficult for models to predict the sharp fall that followed immediately. Also, as is evident, the series is more volatile following the peak, relative to the in-sample, thus making it a difficult period for predictions.
} 
which factors play important roles at each point in time in forecasting gold returns over different horizons of the out-of-sample period, based on time-varying posterior inclusion probabilities. The results shows that the good predictors of the gold prices are the exchange rate, the financial stress index, stock market and the business cycle factors which can be considered as the leaders of the forecasting procession over the forecast periods. Nevertheless, the forecasting prowess of the exchange rate is supreme.

There are benefits from finding the right model that forecasts the gold price returns more accurately than others. Out-of-sample forecasting offers informational advantage for monetary policymakers, hedge fund managers and international portfolio managers which can be used in gauging future inflation, estimating demand for jewellery, discerning investment in precious metals and other commodities and assessing the future movement of the dollar exchange rate. It also provides additional support to the hypothesis that the economic activity, foreign exchange, stocks, interest rates and gold prices are driven by the same information sets of predictors. This lends support to the belief that the world gold market is currently dominated by the U.S. dollar bloc.

The reminder of the paper is organized as follows. Section 2 presents a brief of review of the literature. Section 3 describes the methodology and Section 4 discusses the results. Section 5 concludes the paper.

\section{Review of related literature}

The literature on forecasting changes in the gold returns is not as generous as it is on gold return movement and volatility, and the methods employed in forecasting are not as sophisticated either. Ismail et al. (2009) use the multiple linear regression to predict the future gold prices using a set of economic factors selected based on "a hunch of experts" including Commodity Research Bureau future index, U.S. dollar to euro foreign exchange rate, inflation rate, money supply, S\&P 500 index, Treasury Bill and U.S. nominal effective dollar index. The 
predictions are evaluated relative to the performance of a naïve (random-walk) model. The results show that the considered models achieve higher level of predictive accuracy compared to the naïve model.

Sjaasdat (2008) investigates the theoretical and empirical relationships between the price of gold and the major exchange rates, using forecast error data. The author finds the floating exchange rates among the major currencies to be a major source of price instability in the global gold market since the termination of the Bretton Woods international monetary system. Moreover, since this market is currently dominated by the U.S. dollar bloc, appreciations or depreciations of the dollar would have strong effects on the gold price expressed in other currencies. The author finds support for the hypothesis of market efficiency for the world gold market during the 1991-2004 period. However, he has not found that the major gold producers of the world (Australia, South Africa, and Russia) appear to have any significant influence over the world gold price.

Shafiee and Topal (2010) investigate the relationship between the gold price and key influencing factors such as the oil price and global inflation over the last 40 years. The authors employ the trend reverting jump and dip diffusion model to forecasting natural-resource commodity prices including the price of gold. The model examines the fluctuations of prices in terms of a long-term trend reversion component, a diffusion component and a jump or dip component. The model is then applied to forecast the gold price for the next 10 years. The forecast results show that the gold price is predicted to stay abnormally high up to the end of 2014, after that it would revert to the long-term trend until 2018. Lili and Chengmei (2013) use the Factor-Augmented Vector Auto-regression (FAVAR) model to extract three common factors from the panel data series, representing financial market indices, gold reserves and energy prices, and macroeconomic indicators that affect gold prices. 
Pierdzioch et al. (2014a) employ a real-time (recursive, as also in our case) forecasting approach to examine whether publicly available information on a large set of financial and macroeconomic variables would help to forecast out-of-sample monthly excess returns of gold under uncertainty. This approach also accounts for the possibility that the optimal forecasting model may vary over time. The results show that using forecasts implied by this forecasting approach to set up a simple trading rule does not necessarily lead to a superior performance relative to a buy-and-hold strategy. Pierdzioch et al. (2014b) also find some evidence that the international business cycle has predictive power for gold-price fluctuations. They suggest a behavioral-finance approach to study the quality of out-of-sample forecasts from the perspective of forecasters with potentially asymmetric loss functions.

Baur and Glover (2014) develop and test empirically several models incorporating heterogeneous expectations of agents, specifically fundamentalists and chartists, for the gold market. The empirical evidence shows that both agent types are important in explaining historical gold prices. However, the 10-year bull run of gold in the early 2000s is consistent with the presence of agents extrapolating long-term trends.

On the benefits of forecasting the gold price, Apergis (2014) investigates whether the gold price has a reliable out-of-sample relationship with the Australian dollar/U.S. dollar nominal and real exchange rates using daily and quarterly data, respectively. Using an Error Correction Model (ECM), the empirical results suggest that the out-of-sample predictive ability is strong and robust across short- and long-run horizons, thus offering informational availability for monetary policymakers, hedge fund managers and international portfolio managers. They also provide additional support to the hypothesis that both markets are driven by the same information sets.

In summary, the papers that are most closely related to our paper is that of Pierdzioch et al. (2014a, b), in the sense that those authors look at similar set of predictors, though fewer in numbers and primarily concentrated for the U.S., and also allow for model uncertainty. 
However, unlike the papers by Pierdzioch et al. (2014a, b), we also model for nonlinearity both in- and out-of-sample, in the relationship between gold returns and its predictors by allowing for time-varying coefficients. The recursive (real-time) scheme adopted by Pierdzioch et al. (2014a, b) only allows for nonlinearity over the out-of-sample. And as we show, the constant parameter models, namely the BMA and a special case of DMA where the inclusion of the predictors varies over time only (but not the coefficients), based on recursive estimations over the out-of-sample is easily outperformed by the DMA and DMS. Further, we are able to provide information on the importance of specific predictors, for each forecasting horizon at each point of the out-ofsample based on posterior inclusion probabilities. This forecasting information matters and is helpful because it relates relevant predictors to specific time periods and saves investors from incurring losses and policy-makers from making mistakes at the wrong time by helping them obtain better signals.

\section{Methodology}

The models considered for forecasting the gold price return in this study include the time-varying parameter (TVP) model, the dynamic model averaging (DMA) model, the dynamic model selection (DMS) model, the Bayesian model averaging (BMA) model and a random walk (RW) model.

The time-varying parameter (TVP) models employ state space methods such as the Kalman filter, which is commonly used in empirical macroeconomic research on structural analysis and forecasting. These types of models, however, do not allow predictors to vary over time (Koop and Korobilis, 2012). ${ }^{3}$ If large sets of predictors are used, then the TVP models tend to over-fit in-sample, and therefore have a poor out-of-sample forecasting performance. Even

${ }^{3}$ This section relies heavily on the discussion available in Koop and Korobilis (2012), to the extent that we have also retained the mathematical symbols they used in their equations. 
extensions of these models such as the TVP-VAR models suffer from this same limitation (Koop and Korobilis, 2012). To address these shortcomings in the TVP models, the DMA models present a possible and better alternative.

The dynamic model averaging (DMA) model, as the name implies, averages across various models. To understand the econometric methodology regarding DMA, suppose that we have a set of $K$ models which are characterized by having different subsets of $z_{t}$ as predictors.

Denoting these subsets by $z^{(k)}$ for $k=1, \ldots, K$, our set of models can be written as:

$$
\begin{aligned}
& y_{t}=z_{t}^{(k)} \theta_{t}^{(k)}+\varepsilon_{t}^{(k)} \\
& \theta_{t+1}^{(k)}=\theta_{t}^{(k)}+\eta_{t}^{(k)}
\end{aligned},
$$

where $y_{t}$ is the dependent variable being forecast, i.e., the return of the gold price, $\varepsilon_{t}^{(k)}$ is $N\left(0, H_{t}^{(k)}\right)$ and $\eta_{t}^{(k)}$ is $N\left(0, Q_{t}^{(k)}\right)$. Let $L_{t} \in\{1,2, . ., K\}$ denote which model applies at each time period, the coefficient vector be $\Theta_{t}=\left(\theta_{t}^{(1)^{\prime}}, \ldots, \theta_{t}^{(K)^{\prime}}\right)^{\prime}$ and the output vector be $y^{t}=\left(y_{1}, \ldots, y_{t}\right)^{\prime}$. The fact that we are allowing different models to hold at each point in time and performing model averaging, gives rise to the terminology "dynamic model averaging”. To be precise, when forecasting time $t$ variables using information through time $t-1$, DMA involves calculating the probabilities $\operatorname{Pr}\left(L_{t}=k \mid y^{t-1}\right)$ for the models $k=1, \ldots, K$, and averaging forecasts across those $K$ models, using these probabilities. DMS involves selecting the single model with the highest value for $\operatorname{Pr}\left(L_{t}=k \mid y^{t-1}\right)$ and using this model to forecast. However, there are problems with such a framework, since many of the models can have a large number of parameters, and the computational burden which arises when $K$ is large implies that estimation can take a long time. Thus, a full Bayesian approach to DMA can be quite difficult.

Following Koop and Korobilis (2012), in this paper we use approximations as suggested by Raftery et al., (2010). These approximations involve two parameters for the coefficients and 
the models, $\lambda$ and $\alpha$, which Raftery et al., (2010) refer to as the forgetting factors, and fix them to numbers slightly below one.

To explain the role of these forgetting factors, first consider the standard state space model below for $t=1 \ldots . T$ :

$$
\begin{aligned}
& y_{t}=z_{t} \theta_{t}+\varepsilon_{t} \\
& \theta_{t}=\theta_{t-1}+\eta_{t}
\end{aligned}
$$

In our case, the output vector $y_{t}$ is the return of the gold price, the vector $z_{t}=\left[1, x_{t-1}, y_{t-1}, \ldots, y_{t-p}\right]$ is an $1 \times m$ vector of predictors for the return of the gold price which also includes an intercept and lags of the dependent variable, the vector $\theta_{t}=\left[f_{t-1}, \beta_{t-1}, \gamma_{t-1}, \ldots, \gamma_{t-p}\right]$ is an $m \times 1$ vector of coefficients (states), and $\varepsilon_{t} \sim N\left(0, H_{t}\right)$ and $\eta_{t} \sim N\left(0, Q_{t}\right)$ are the errors which are assumed to be mutually independent at all leads and lags. For given values of the variance-covariance matrices $H_{t}$ and $Q_{t}$, the standard filtering and smoothing results can be used to carry out recursive estimation or forecasting. Kalman filtering begins with the result that

$$
\theta_{t-1} \mid y^{t-1}: N\left(\hat{\theta}_{t-1}, \sum_{t-1} \mid t-1\right)
$$

where formulae for $\hat{\theta}_{t-1}$ and $\sum_{t-1 \mid t-1}$ which are defined below are standard. Note here that these formulae depend one variance-covariance matrices $H_{t}$ and $Q_{t}$. for the errors. Then Kalman filtering proceeds, using:

$$
\theta_{t} \mid y^{t-1} \square N\left(\hat{\theta}_{t-1}, \Sigma_{\mathrm{t} \mid t-1}\right),
$$

where

$$
\sum_{t \mid t-1}=\sum_{t-1} \mid t-1+Q_{t} .
$$

Raftery et al. (2010) note that things simplify substantially if this latter equation is replaced by: 


$$
\sum_{t \mid t-1}=\frac{1}{\lambda} \sum_{t-1 \mid t-1}
$$

or, equivalently, $Q_{t}=\left(1-\lambda^{-1}\right) \sum_{t-1 \mid t-1}$ where $0<\lambda \leq 1$. The term "forgetting factor" is suggested by the fact that this specification implies that observations $j$ periods in the past have weight $\lambda^{j}$. An alternative interpretation is that it implies an effective window size of $1 /(1-\lambda)$. In the literature, it is common to choose a value of $\lambda$ near one, which suggests a gradual evolution of coefficients. Raftery et al. (2010) set $\lambda=0.99$. For monthly macroeconomic data, this suggests observations five (one) years ago receive approximately 50\% (90\%) as much weight as last period's observation. This would be consistent with fairly stable models where the coefficient change is gradual. However, setting $\lambda=0.95$, suggests that observations five years ago receive only $5 \%$ as much weight as last period's observation. This would suggest substantial parameter instability with rapid change in coefficients.

To obtain the unconditional prediction, we follow the suggestion of Raftery et al. (2010) and as used in Koop and Korobilis (2012), based on a forgetting factor $\alpha$ for the state equation for the models which in turn, is, comparable to the forgetting factor $\lambda$ used with the state equation for the parameters. The derivation of Kalman filtering ideas begins with (4). For DMA, the result is ${ }^{4}$

$$
\begin{aligned}
& P\left(\Theta_{t-1}, L_{t-1} \mid y^{t-1}\right)=\sum_{k=1}^{K} P\left(\theta_{t-1}^{(k)} \mid L_{t-1}=k, y^{t-1}\right) \operatorname{Pr}\left(L_{t-1}=k \mid y^{t-1}\right) \\
& \text { where } P\left(\theta_{t-1}^{(k)} \mid L_{t-1}=k, y^{t-1}\right) \text { is given by } \Theta_{t-1 \mid L_{t-1}}=k, y^{t-1} \square N\left(\hat{\theta}_{t-1}^{(k)}, \sum_{\mathrm{t}-1 \mid \mathrm{t}-1}^{(\mathrm{k})}\right) \text {. To }
\end{aligned}
$$

simplify notation, let $\pi_{t \mid s, l}=\operatorname{Pr}\left(L_{t}=l \mid y^{s}\right)$ and thus, the final term on the right hand side

\footnotetext{
${ }^{4}$ For technical details on Kalman filtering under conditional prediction for both single and multi-model non-DMA cases see Koop and Korobilis (2012).
} 


$$
\text { of (7) is } \pi_{t-1 \mid t-1, k} \text {. The model prediction equation is given as } \pi_{t \mid t-1, k}=\frac{\pi_{t-1 \mid t-1, k}^{\alpha}}{\sum_{l=1}^{K} \pi_{t-1 \mid t-1, l}^{\alpha}} \text {, }
$$

where $0<\alpha \leq 1$ is another foregetting factor similar to $\lambda$. Its interpretation is similar to $\lambda$ but in terms of the evolution of models rather than the evolution of parameters. If $\alpha=0.99$ (our benchmark value and also the value used by Raftery et al., 2010) and we are using monthly data, the forecast performance five years ago receives $50 \%$ as much weight as the forecast performance of last period, while the forecast performance one year ago receives about $90 \%$ as much weight as last month's forecast performance. If $\alpha=0.95$, then forecast performance five years ago receives only about $5 \%$ as much weight as last period's forecast performance. Comparable to those of the updating equation in the Kalman filter, we have a model updating equation of:

$$
\pi_{t \mid t, k}=\frac{\pi_{t \mid t-1, k P k}\left(y_{t} \mid y^{t-1}\right)}{\sum_{l=1}^{K} \pi_{t \mid t-1, l P l}\left(y_{t} \mid y^{t-1}\right)}
$$

where $p_{l}\left(y_{t} \mid y^{t-1}\right)$ is the predictive density for model $l$ (which is simply a Normal density) evaluated at $y_{t}$. Recursive forecasting can be done by averaging over predictive results for every model using $\pi_{t \mid t-1, k}$. Therefore, DMA point predictions are given by:

$$
E\left(y_{t} \mid y^{t-1}\right)=\sum_{k=1}^{K} \pi_{t \mid t-1, k} z_{t}^{(k)} \hat{\theta}_{t-1}^{(k)}
$$

DMS proceeds by selecting the single model with the highest value for $\pi_{t \mid t-1, k}$ at each point in time and simply using it for forecasting. Note also that, if $\alpha=1$, then $\pi_{t \mid t-1, k}$ is simply proportional to the marginal likelihood using data through time $t-1$, and yields the standard approaches to BMA. If we also set $\lambda=1$, then we obtain BMA using conventional linear forecasting models with no time variations in coefficients. In our forecast comparison exercise, 
we include BMA in our set of alternative forecasting procedures and implement this by setting $\alpha=\lambda=1 .^{5}$

\section{Data description}

As indicated earlier, the data include six groups of predictors of the gold return. These predictors are categorized as the business cycle factor: the nominal factor; the interest rate factor; the commodity factor, the exchange rate market; and the stock price factor. In addition to these factors, we added the U.S. economic uncertainty and the Kansas City Fed's financial stress index to capture the impacts of policy uncertainty and anxiety in the economy. Altogether, we have 28 predictors which are then categorized as above. Each factor is created based on the first recursive principal component of the variables in that group. The business cycle factor, for example, is represented by the industrial production for the United States, the European Union (EU), China and Japan. An increase in this factor implies increases in the demand for jewelry which makes up more than $65 \%$ of total gold demand. The same description applies to the other factor. However, for robustness check, we also implemented the recursive principal component analysis (PCA) using all the 28 predictors without categorization. In this case we extract four principal components as suggested by the Bai and $\mathrm{Ng}(2002)$ tests.

One of the biggest advantages of using the principal component in our study is that it greatly reduces the number of parameters to estimate. This issue becomes crucial when we allow those parameters to vary over time. Moreover, using the recursive version allows for parameter updating as new data becomes available and also accounts for time variation. Based on the data availability, especially the availability of the China data, the sample period is January 1992 November 2013 (263 monthly observations) and the detailed list and the sources of the variables are available in Table A1 in the Data Appendix. Note that, due to data transformation to ensure

\footnotetext{
${ }^{5}$ Further details on the DMA and DMS methods and implementation can be obtained from Koop and Korobilis (2011, 2012).
} 
stationarity, which is required for both extracting factors and convergence issues in time-varying models, our effective sample starts from February, 1992, i.e., 1992:02, and goes until 2013:11. Our sample is divided into in-sample (1992:01 to 1999:09) and out-of-sample (1999:10 to 2013:11), for the purpose of the recursive PCA and forecasting. For the PCA we first estimated the model using the entire in-sample period data, then subsequently solved the out-of-sample recursively.

The recursive PCA reveals that the first principal component in each group explains: 38 percent of the total variation in the business cycle group, 30 percent in the nominal group, 61 percent in the interest rate group, 69 percent in the commodity price group, 74 percent in the exchange rate group, and 73 percent in the stock price group. The common factors are extracted based on the their economic relevance. ${ }^{6}$

Kansas City Federal Reserve Bank's financial stress index (KCFSI), which is not part of the principal components analysis, measures the degree of financial stress in the financial markets and is constructed from 18 data financial series, where each of these series captures some aspect of financial stress. This index is accessed from the Federal Reserve Bank of Saint Louis's database. The U.S. economic policy uncertainty index (EPU) ${ }^{7}$ is introduced by Baker et al. (2012). It is constructed from three types of underlying components. The first component quantifies newspaper coverage of policy-related economic uncertainty. The second reflects the number of federal tax code provisions set to expire in future years. The third uses disagreement among economic forecasters as a proxy for uncertainty. A weighted normalized index is created from those three components. This index spiked on October 3, 2008 which is the depth of the GFC.5.

\footnotetext{
${ }^{6}$ One could include more than one principal component in each group. For example, adding a second principal component in the business cycle category will improve the explanatory power by 24 percentage points. However, it will be difficult to interpret or to give economic meanings. To give better economic meaning to the factors, we therefore extract one common factor from each group.

${ }^{7}$ See http://www.policyuncertainty.com
} 


\section{Empirical Results}

We present the empirical results in two sub-sections, using DMA and DMS as in Koop and Korobilis (2011), i.e., by setting $\alpha=0.99$ and $\lambda=0.99^{8}$, a non-informative prior over the models $\left(\pi_{0 \mid 0, k}=1 / K\right.$ for $\left.k=1, \ldots, K\right)$ and a relatively diffuse prior for the initial state conditions: $\theta_{0}^{(k)} \sim N(0,100)$ for $k=1, \ldots, K$. The first sub-section provides evidence of which variables are good predictors of the gold price returns. In the second sub-section, we compare the forecast performance of the DMA to those of a number of alternative forecasting models nested in the DMA, including the BMA, TVP and random walk models. The considered forecast horizons are: one $(b=1)$, three $(b=3)$, six $(b=6)$, nine $(h=9)$ and $12(b=12)$ months. All of the models include an intercept and a zero lag of the dependent variable as chosen by the Schwarz information criterion.

\subsection{Good predictors for the gold price returns}

One of the largest potential advantages of using the DMA framework is that it allows the forecasting model to change over time, i.e. the model parameters may change as well as the set of predictors. Given the difficulty in the literature in explaining the gold price return movements, we include a set of 8 possible predictors (excluding the intercept) as categorized and listed in the Data Appendix, hence we have 256 possible models to choose from. Similar analysis with the four factors extracted from all the 28 predictors uncategorized, yields 16 possible models to choose from.

Turning to results on the main predictors as selected by the DMA analysis for gold for every forecast horizon, $b=1, b=3, b=6, b=9$ and $h=12$, over the out-of-sample period 1999:10 to

\footnotetext{
${ }^{8}$ Note that, one could choose values for $\lambda$ and $\alpha$ based on the forecast performance as in Grassi and Santucci de Magistris (2013), but this would bias our results in favour of DMA and thus is not a valid procedure for out-ofsample forecasting (Koop and Korobilis, 2011). Alternatively, when forecasting at time $\tau$, we could consider a grid of values for $\lambda$ and $\alpha$, and select the value which yielded the highest value for the marginal likelihood or an information criterion, which essentially amounts to treating $\lambda$ and $\alpha$ as unknown parameters. However, this would greatly add to the computational burden; so much so that it might be impossible to do forecasting in real time (Koop and Korobilis, 2011). Hence, we follow Koop and Korobilis $(2011,2012)$ and simply select values for the forgetting factors.
} 

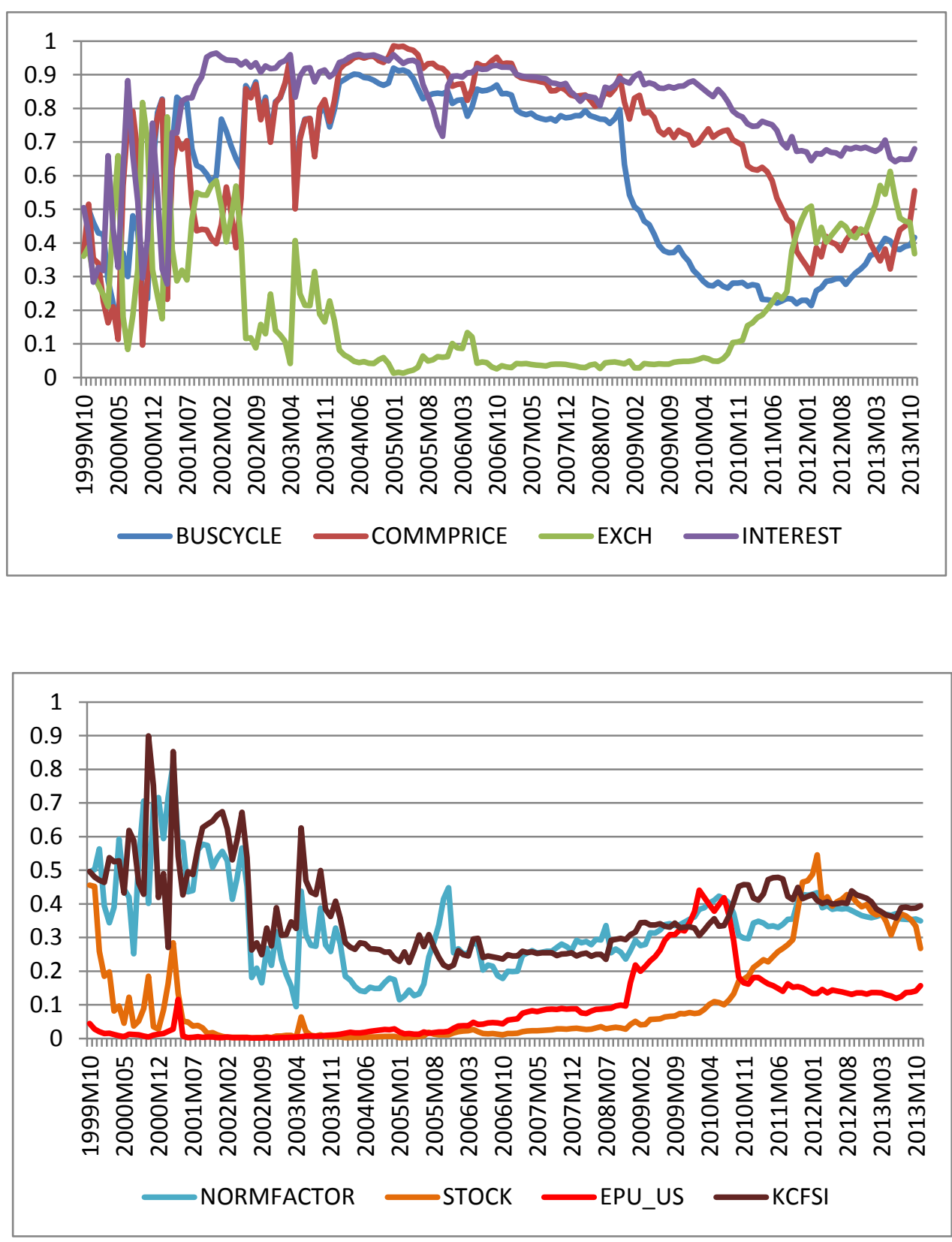

Figure 2: Posterior inclusion probabilities of predictors $(b=1)$ 

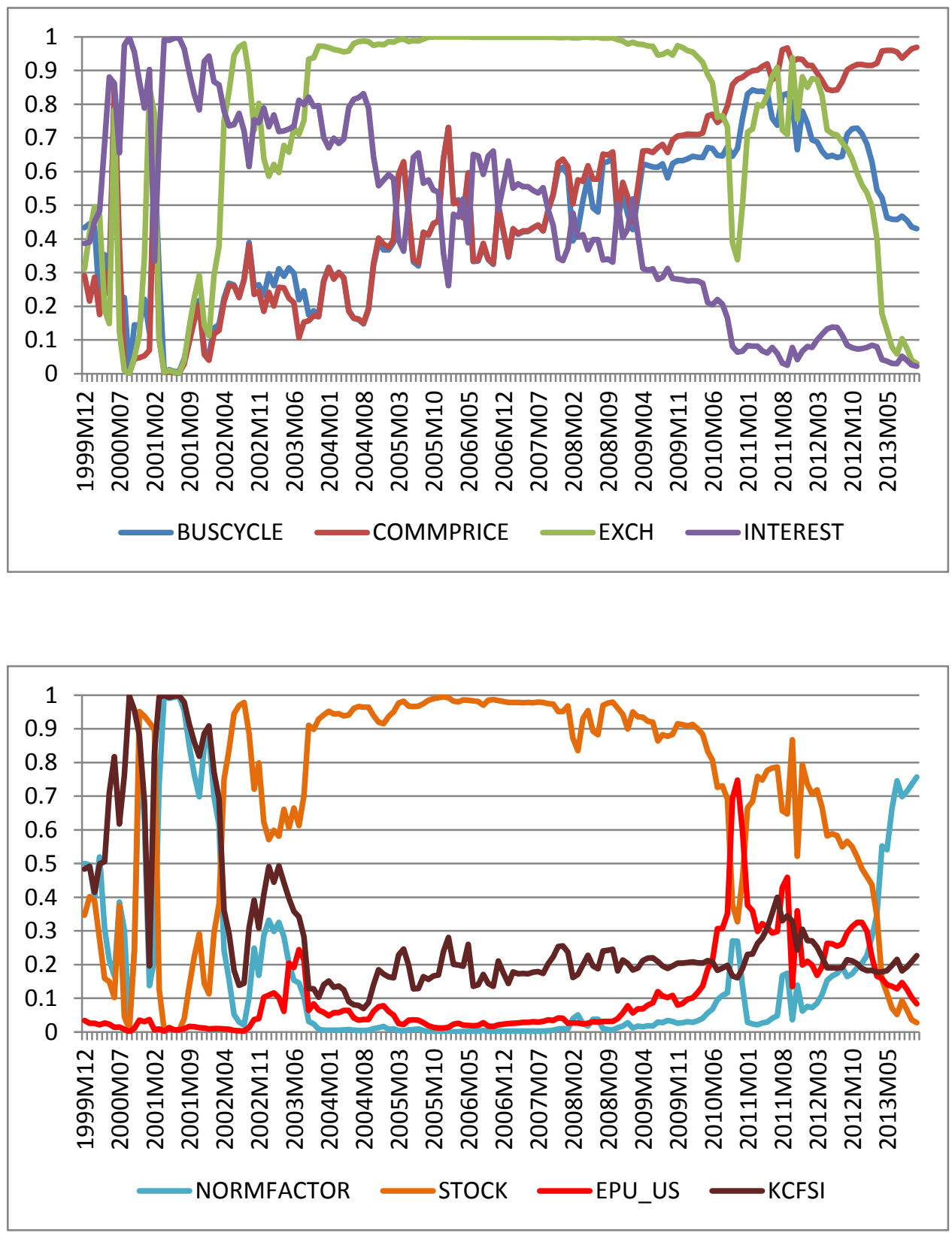

Figure 3: Posterior inclusion probabilities of predictors $(b=3)$ 

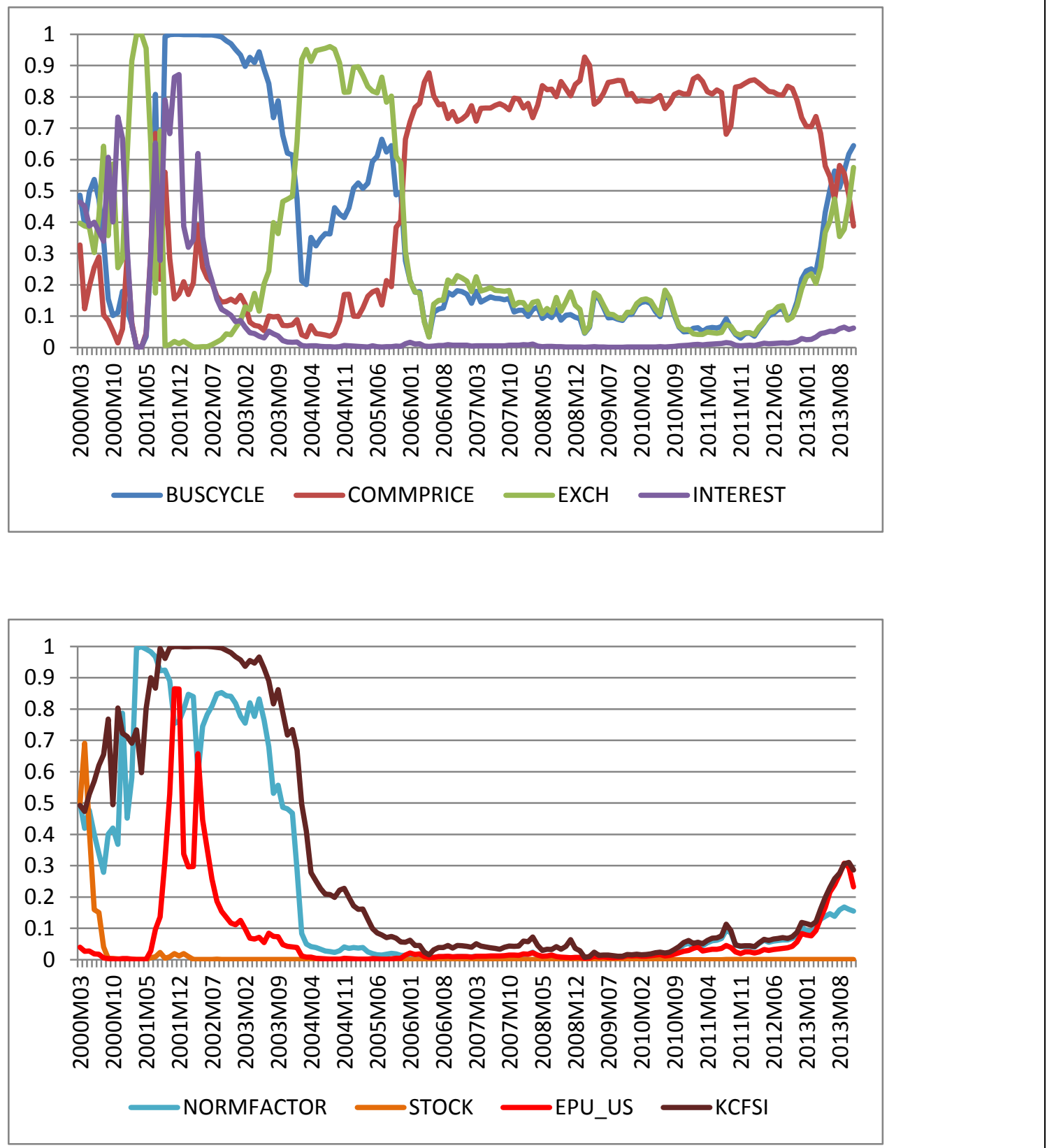

Figure 4: Posterior inclusion probabilities of predictors $(b=6)$ 

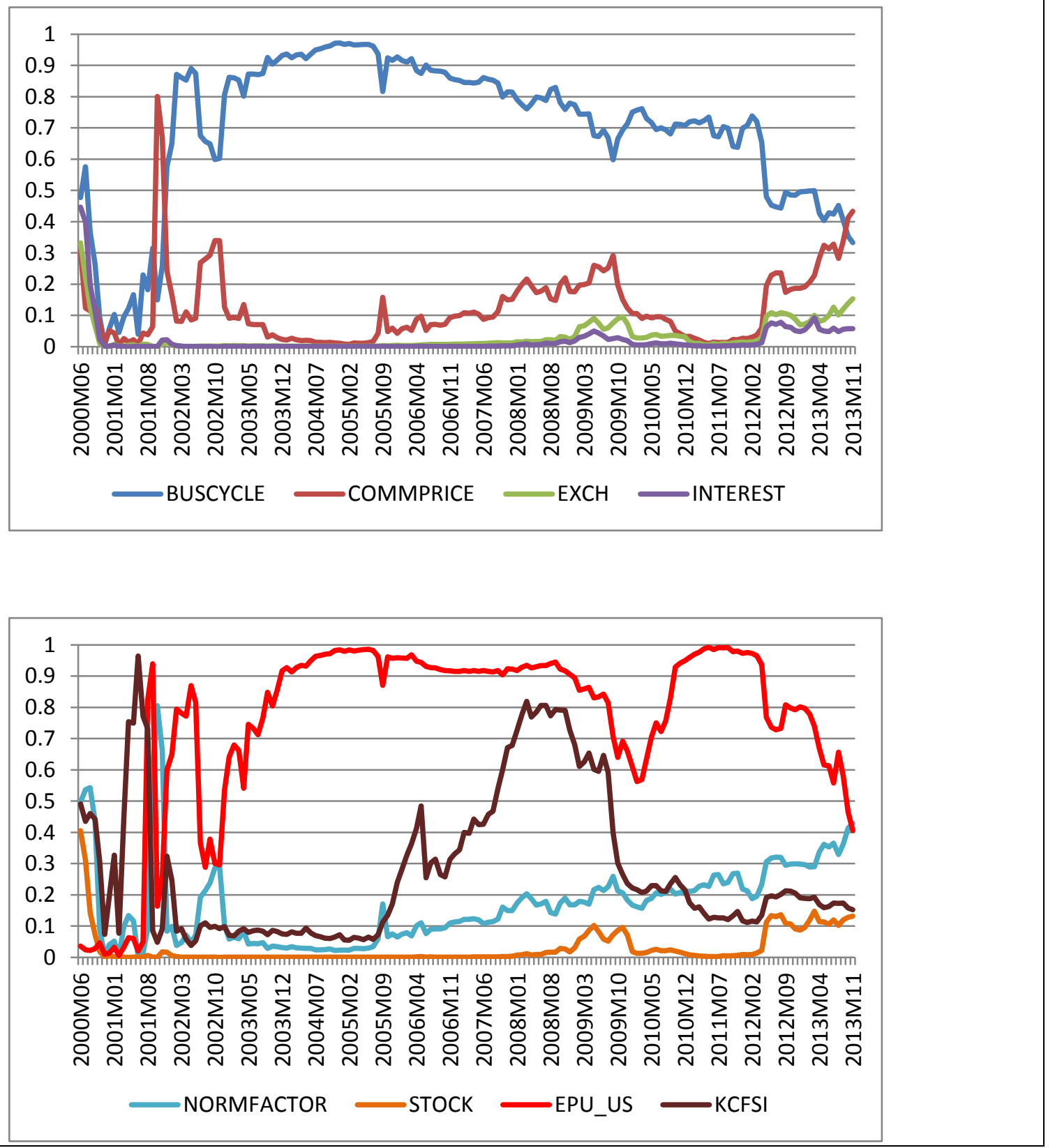

Figure 5: Posterior inclusion probabilities of predictors $(b=9)$ 

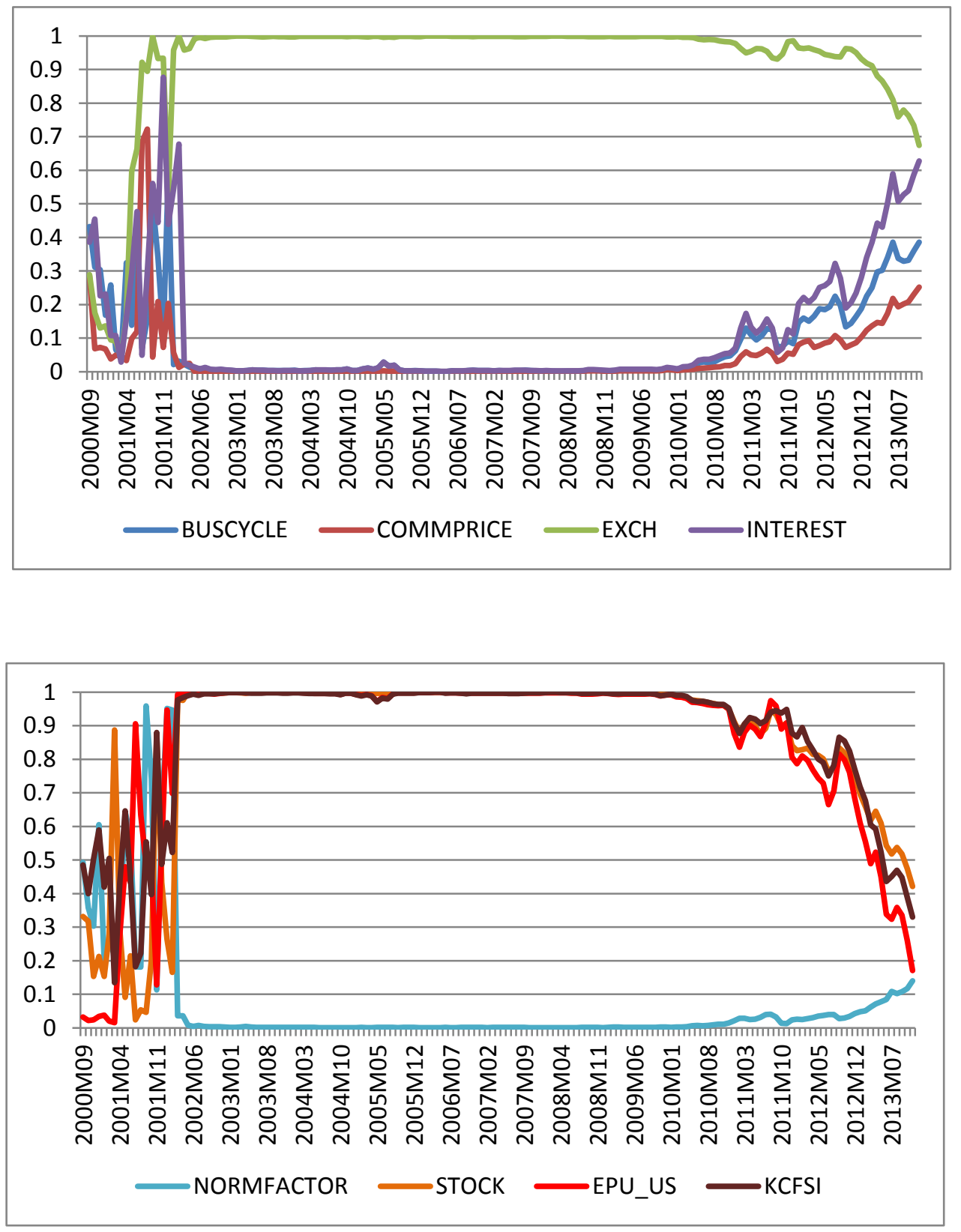

Figure 6: Posterior inclusion probabilities of predictors $(b=12)$

2013:11. The posterior probabilities of inclusion of each predictor for the different forecast horizons, which shed light on their predictive abilities, are presented in Figures 2 to 6. ${ }^{9}$ It appears that the good predictors of gold price returns vary over time and over the forecast horizons.

\footnotetext{
${ }^{9}$ We do not present the corresponding results for the four factors extracted from the uncategorized 28 predictors. The results based on the estimated marginal $\mathrm{R}^{2}$ obatined by regressing each 28 variables on each of the four principal components do not clearly show which variable or category of variables has more explanatory power over the others. To be precise, the results are mixed, thus making the economic interpretation of the four different principal components complex.
} 
Particularly, three periods are striking namely pre-2005, 2005-2009 and post 2009. It must be noted that the middle period includes the heart of the boom for commodities and the Great Recession period, while the third is the post Great Recession or recovery period.

Prior to 2005, the exchange rate factor $(E X C H)$ and the interest rate (INTEREST) show strong predictive power for all the horizons except for $b=9$. The business cycle factor (BUSCYCLE) comes through strongly for all horizons but $b=3$ and $b=12$. We observe the US economic policy uncertainty (EPU_US) playing a strong predictive role for $b=6, b=9$ and $b=12$. The nominal factor (NORMFAC) shows strong predictive power at essenatially all horizons except that this was shortlived at horizons 3 and 9 while the stock market factor (STOCK) possesses strong predictive power for $b=3$ and 12 . The commodity prices factor (COMMPRICE) comes through strongly and persistently for $b=1$ only. The Kansas City Fed' Financial Stress Index (KCFSI) shows strong predictive power for all horizons except for $\mathrm{h}=9$ where the predictive ability was only for a short period. In sum, in the pre-2005 period the exchange rate, the financial stress index, interest rate, and norminal factors are the leaders of the forecasting procession.

Between 2005 and 2009, the same pattern is observed in that different predictors emerged as best for different forecast horizons. At $h=1$, INTEREST, COMMPRICE and BUSCYCLE factors took the lead. At $b=3$, it is EXCH and STOCK while at $b=6$, only COMMPRICE has strong predictive power for gold returns. At $b=9$ the best predictors are BUSCYCLE, EPU and KCFSI while EXCH, STOCK, EPU_US and KCFSI comes through strongly at $b=12$. We note that it is somewhat difficult to select a single predictor here as the overall best. The least we can say is that only INTEREST did not feature as a very good predictor in this era since it appears as a strong predictor for one horizon while the rest are strong for two horizons. . For the post 2009 period, we observe $\mathrm{EXCH}$ maintaining the same predictive roles as in the previous subperiod. STOCK has also consistently maintained its predictive power for $b=3$ and 12 across all the three sub-periods. INTEREST comes through strongly for $h=1$ and 12 , while EPU_US 
comes through strongly for $h=3,9$ and 12 . COMMPRICE plays a very strong predictive role for $b=1,3$ and 6.NORMFAC and KCFSI have predictive power for only $b=3$ and $b=12$, respectively. BUSCYCLE shows strong predictive power for $b=3$ and 9.

Overall, all the predictors show strong predictive power at one time or another though at varying magnitudes, while EXCH and KCFSI appear to be strong at almost all horizons and subperiods. However, the forecasting prowess of the exchange rate is supreme. Gold as a means of exchange and store of value makes it akin to money. Therefore, the performance of exchange rate in predicting gold returns is not surprising given that exchange rates incorporate expectations regarding fundamentals with respect to future discounted values. This findings supports the view that exchange rate is a forward looking price which embodies information about future variables (Engel and West, 2005; Bacchetta and van Wincoop, 2006).

\section{2. Model forecasting comparison}

To analyze the forecast performance of each model, we use the mean squared forecast error (MSFE) in percentages, which is available for all the models. Note that, the preferred method for the Bayesian forecast comparisons is the sum of the log predictive likelihoods, involving the entire predictive distribution. For a discussion on predictive likelihoods, refer to Gweke and Amisano (2007) as a representative source. The predictive likelihood is the predictive density for $y_{t}$, given data through time $t-1$, evaluated at the actual outcome. Since we use the direct method of forecasting, the log predictive density for the $h$-step ahead forecast is an obvious extension of the formula for the one-step ahead predictive density in model $l$ as denoted by $p_{l}\left(y_{t} \mid y^{t-1}\right)$ and described in Section 3. We use the sum of log predictive likelihoods for the forecast evaluation of the Bayesian models, with the sum beginning in the tenth month of 1999 up to the eleventh month of 2013 of the out-of-sample period. MSFE's are reported over the same period.

We present results for the following alternative forecasting models: 
(1) Forecasts using Dynamic Model Averaging (DMA) with $\alpha=\lambda=0.99$.

(2) Forecasts using Dynamic Model Selection (DMS) with $\alpha=\lambda=0.99$.

(3) Forecasts using all the variables in a single model with time varying parameters (TVP) (100\% of the prior weight is attached to the model with all the variables in this special case of DMA, with all other modeling choices being identical including $\lambda=0.99$ ).

(4) Forecasts using DMA, but the coefficients do not vary over time (a special case of DMA where $\lambda=1$ and $\alpha=0.99)$.

(5) Forecasts using Bayesian Model Averaging (BMA: a special case of DMA where $\alpha=\lambda=1)$

(6) Forecasts using the random walk (RW).

Model (1) applies the dynamic model averaging (DMA), using the benchmark values $\alpha=\lambda=0.99$. This allows not only for the parameters to change over time, but also for the set of predictors. As stated earlier, values of 0.99 are consistent with fairly stable models with gradual coefficient changing over time, where forecasts are averages across models using the associated probabilities calculated as $\operatorname{Pr}\left(L_{t}=k\left(y^{t-1}\right)\right.$. Model (2), where dynamic model selection (DMS) is used, involves selecting the single model with the highest probability and using this to forecast. Model (3) is a special case of Model (1) where all predictors are included for all time periods, but parameter values are allowed to change. Model (4) is a constant parameter model but the model evolution is allowed, while in Model (5), through setting both forgetting factors to one, we obtain the Bayesian model averaging (BMA) which uses conventional linear forecasting models with no time variations in the coefficients. Understandably, the random walk (RW) model is non-Bayesian, which means that there is no predictive likelihood for the model.

Table 1 presents the results from our forecasting exercise for the gold price return for five forecasting horizons, namely 1-month-ahead (Horizon 1) , 3-months-ahead (Horizon 3), 6months-ahead (Horizon 6), 9-months-ahead (Horizon 9), and 12-months-ahead (Horizon 12). We present the results of the forecast performance of the various models based on the first 
factor from each of the listed categorized predictors in the appendix and the four factors extracted from the uncategorized 28 predictors. ${ }^{10}$ From the results in Table 1, it is clear that the benchmark DMA (i.e the case where $\alpha=\lambda=0.99$ ) and DMS forecast generally well, with DMS being the best overall. The sum of the log predictive likelihoods clearly shows that DMS performs better across all forecast horizons. This result also carries over to the MSFE. These

Table 1: Comparing different forecasting methods for gold price returns

\begin{tabular}{|c|c|c|c|c|}
\hline \multicolumn{2}{|c|}{ Uncategorized predictors } & \multicolumn{3}{|c|}{ Categorized predictors } \\
\hline Forecast Method & $\begin{array}{l}\text { Sum of Log } \\
\text { predictive } \\
\text { likelihoods }\end{array}$ & MSFE & $\begin{array}{l}\text { Sum of Log } \\
\text { predictive } \\
\text { likelihoods }\end{array}$ & MSFE \\
\hline & $b=1$ & & & \\
\hline DMA $(\alpha=0.99, \lambda=0.99)$ & -472.401 & 19.017 & -477.084 & 22.314 \\
\hline DMS $(\alpha=0.99, \lambda=0.99$ & -464.281 & 15.493 & -466.466 & 17.272 \\
\hline $\operatorname{TVP}(\alpha=1, \lambda=0.99)$ & -473.485 & 18.988 & -479.269 & 22.345 \\
\hline $\operatorname{DMA}(\alpha=0.99, \lambda=1)$ & -475.102 & 18.912 & -477.393 & 22.384 \\
\hline BMA (DMA with $\alpha=\lambda=1$ ) & -476.511 & 19.039 & -479.966 & 22.428 \\
\hline \multirow[t]{2}{*}{ Random walk } & - & 31.253 & - & 31.253 \\
\hline & $b=3$ & & & \\
\hline DMA $(\alpha=0.99, \lambda=0.99)$ & -520.198 & 24.915 & -499.462 & 25.396 \\
\hline DMS $(\alpha=0.99, \lambda=0.99$ & -521.734 & 23.387 & -496.362 & 22.682 \\
\hline $\operatorname{TVP}(\alpha=1, \lambda=0.99)$ & -520.518 & 24.869 & -507.264 & 26.687 \\
\hline $\operatorname{DMA}(\alpha=0.99, \lambda=1)$ & -521.995 & 28.029 & -503.095 & 26.583 \\
\hline BMA (DMA with $\alpha=\lambda=1$ ) & -523.214 & 28.287 & -510.564 & 27.570 \\
\hline \multirow[t]{2}{*}{ Random walk } & - & 29.839 & - & 29.839 \\
\hline & $b=6$ & & & \\
\hline DMA $(\alpha=0.99, \lambda=0.99)$ & -483.112 & 20.594 & -486.778 & 25.216 \\
\hline DMS $(\alpha=0.99, \lambda=0.99$ & -478.601 & 18.716 & -476.728 & 23.699 \\
\hline $\operatorname{TVP}(\alpha=1, \lambda=0.99)$ & -487.685 & 21.492 & -491.037 & 26.122 \\
\hline $\operatorname{DMA}(\alpha=0.99, \lambda=1)$ & -486.355 & 21.922 & -488.785 & 25.623 \\
\hline BMA (DMA with $\alpha=\lambda=1$ ) & -492.449 & 23.028 & -493.725 & 26.638 \\
\hline \multirow[t]{2}{*}{ Random walk } & - & 33.123 & - & 33.123 \\
\hline & $b=9$ & & & \\
\hline DMA $(\alpha=0.99, \lambda=0.99)$ & -461.742 & 17.131 & -458.384 & 18.465 \\
\hline DMS $(\alpha=0.99, \lambda=0.99$ & -460.592 & 16.778 & -453.518 & 17.403 \\
\hline $\operatorname{TVP}(\alpha=1, \lambda=0.99)$ & -465.618 & 17.164 & -463.974 & 18.866 \\
\hline $\operatorname{DMA}(\alpha=0.99, \lambda=1)$ & -466.902 & 17.259 & -460.026 & 18.693 \\
\hline BMA (DMA with $\alpha=\lambda=1$ ) & -468.051 & 17.287 & -463.828 & 19.224 \\
\hline \multirow[t]{2}{*}{ Random walk } & - & 33.480 & - & 33.480 \\
\hline & $b=12$ & & & \\
\hline DMA & -477.579 & 20.232 & -441.460 & 17.925 \\
\hline DMS & -474.858 & 18.684 & -437.752 & 17.154 \\
\hline $\operatorname{TVP}(\alpha=1)$ & -479.182 & 20.159 & -444.038 & 18.426 \\
\hline $\operatorname{DMA}(\lambda=1)$ & -477.198 & 20.047 & -440.796 & 18.028 \\
\hline BMA (DMA with $\alpha=\lambda=1$ ) & -478.633 & 20.002 & -444.578 & 18.777 \\
\hline Random walk & - & 33.476 & - & 33.476 \\
\hline
\end{tabular}

${ }^{10}$ We conducted out-of-sample forecasts from each of the 28 observed predictors, relative to the random walk model, using OLS-based recursive estimation over the out-of-sample period. The Theil's U statistic is presented in the table below. Theil's $U$ values less than one indicates the superior forecast performance of the unrestricted model (model with a predictor and constant) over the restricted random walk model. The results show that in general the individual regressions did not perform better than the random walk model. This, to some extent, illustrates the importance of combining information based on factors, and more importantly allows us to get around the technical issue of estimating the large number of models at each point in time. 
findings are robust to different specifications of the recursive PCA model. In other words, whether all the 28 variables are included at the same time in the recursive PCA or categorized, the benchmark DMA and DMS have better predictive power for gold returns than the rest of the models in general. Although DMS and DMA can be interpreted as doing the shrinkage in different ways, DMS shrinks the contribution of all models except one towards zero. This additional shrinkage appears to have given DMS additional benefits over DMA. This finding is consistent with forecasts obtained by Koop and Korobilis (2012) for inflation and Gupta et al. (2014) for China's foreign exchange reserves using similar models. Both the sum of the log predictive likelihoods as well as the MSFE indicates that using a TVP model for forecasting results in poorer forecasting performance, relative to the benchmark DMA and DMS. The poor forecast performance of the TVP model compared to DMA and DMS is an indication that the shrinkage provided by the latter models is of great value in forecasting. Also note that, the predictors matter in forecasting, since all the models that include the predictors outperforms the RW model using the 1999:10 to 2013:11 out-of-sample period. ${ }^{11}$ However, to obtain best forecast performances, one not only needs to include information from the fundamentals, but also allows for model evolution and parameter evolution as carried out by the DMA and DMS. Interestingly though, based on the results provided by the predictive likelihoods, we see that most of the improvements in forecast performance found by DMA or DMS are due to model evolution rather than parameter evolution, since DMA and DMS perform better than the DMA model with $\lambda$ set equal to 1 (implying the model is not allowed to change here). It can therefore be concluded that allowing for model uncertainty and not only parameter uncertainty, improves the forecasting performance of these models.

\footnotetext{
11 Although we had previously stated the reason for choosing 1999:10 to 2013:11 as the out-of-sample, as a robustness check, we also conducted the Bai and Perron (2003) multiple structural break tests on the gold returns regressed on a constant, and found that there is only one break in period 2001:5. Hence, we redid the estimation with an out-of-sample period starting in 2001:5 till 2013:11. Our basic conclusions of the superiority of the DMS and DMA relative to the random walk and other models continue to hold. These results are available from the authors upon request.
} 


\section{Conclusion}

Given the difficulty in the literature in forecasting the gold price returns, this study provides evidence of which variables are good predictors of those returns from the list of the six potential factors extracted from a recursive PCA and two individual variables. Then it compares the forecast performance of the DMA model to those of a number of alternative forecasting models nested in DMA, including the BMA and the TVP model as well the random walk model. One of the important advantages of DMA is that it allows the model parameters and the set of predictors to change over time. The forecast horizons are one, three, six, nine and twelve months. The performance of the good predictors of the gold returns varies over time and over the forecast horizons, highlighting the importance of differences in three periods. The first period ranges from 1992 to 2005, which includes three major wars, steady increases in gold prices and part of the commodity boom. The second period covers the years 2005-2009 which includes the last part of the commodity boom and the Great Recession. The third is the post 2009 period which includes the recovery and relative declines in gold prices.

The results attest to the strong predictive power of the exchange rates in forecasting the gold returns over most forecast horizons under the three time periods. This finding is robust to alternative specification of the PCA which involves using all twenty eight variables without categorizing them into six categories and two individual variables. Gold is a means of exchange and store of value. Exchange rate rates incorporate expectations regarding fundamentals in terms of future discounted values. This explains why exchange rate performed well in predicting gold returns.. Two other financial variables with good predictive power include the interest rate factor and the financial stress index (FSI). These variables play a good role in predicting the gold price returns particularly during the first two time periods, the pre-2005 and the 2005-2009 periods. The economic activity factor has a good predictive power that is more pronounced in the first two time periods than in the third period which is the recovery period. This may reflect the low economic growth that did not make economic activity a strong predictor in this period. There is 
also clear evidence that the stock market has good predictive power in the third and twelve horizons across all the three sub-periods, in addition to that possessed by the exchange rate factor..

Moreover, to obtain the best forecast performance, one not only needs to include information from the fundamentals, but also allows for model evolution and parameter evolution as supported by the DMA and DMS. However, the empirical evidence support DMS over DMA. In sum, allowing for model uncertainty and not only parameter uncertainty, improves the forecasting performance of the models.

The major conclusion of this study is that financial variables have stronger predictive power for gold returns. This is particularly so with exchange rate, the financial stress index and the stock market. Our results underscore the importance of increasing financialization of commodities including gold. This conclusion became evident from the important performance of models that takes into consideration parameter and model uncertainties. The out-of-sample forecast results in this study has some policy implications as it offers informational advantage for monetary policymakers, hedge fund managers and international portfolio managers which can be used in gauging future inflation, estimating demand for jewellery, discerning investment in precious metals and other commodities and assessing the future movement of the dollar exchange rate.

\section{References}

Apergis, N. (2014). Can gold prices forecast the Australian dollar movements? International Review of Economics \& Finance 29, 75-82.

Bacchetta, P. and van Wincoop, E. (2006). Can information heterogeneity explain the exchange rate determination puzzle? American Economic Review 96(3), 555-76.

Bai, J. and Ng, S. (2002). Determining the number of factors in approximate factor models. Econometrica, 70(1), 191-221.

Bai, J. and Perron, P. (2003). Computation and analysis of multiple structural change models. Journal of Applied Econometrics, 18(1), 1-22. 
Baker, S. R., Bloom, N. and Davis, S. J. (2012). Measuring economic policy uncertainty. Working Paper, Stanford University.

Baur, D. G. and Glover, K. J. (2014). Heterogeneous expectations in the gold market: Specification and estimation. Journal of Economic Dynamics and Control 40, 116-133.

Baur, D.G. and Lucey, B. M. (2010). Is Gold a Hedge or a Safe Haven? An analysis of stocks, bonds and gold. Financial Review, 45(2), 217-229.

Baur, D.G. and McDermott, T.K.J. (2012). Safe haven assets and investor behaviour under uncertainty. IIIS Discussion Paper No. 392.

https://www.tcd.ie/iiis/documents/discussion/pdfs/iiisdp392.pdf.

Baur, D. G. and McDermott, T. K. J. (2010). Is gold a safe haven? International evidence. Journal of Banking \& Finance, 34(8), 1886 - 1898.

Capie, F., Mills, T. C. and Wood, G. (2005). Gold as a hedge against the dollar, Journal of International Financial Markets, Institutions and Money 15, 343-352.

Ciner, C., Gurdgiev, C. and Lucey, B. M. (2013). Hedges and safe havens: An examination of stocks, bonds, gold, oil and exchange rates. International Review of Financial Analysis 29, 202-211.

Engel, C. and West, K.D. (2005). Exchange rates and fundamentals. Journal of Political Economy 113(3), 485-517.

Geweke, J. and Amisano, G. (2011). Hierarchical Markov Normal Mixture Models with Applications to Financial Asset Returns. Journal of Applied Econometrics 26, 1-29.

Ghazali, M.F., Lean, H-H. and Bahari, Z. (2013). Is gold a hedge or a safe haven? An empirical Evidence of gold and stocks in Malaysia. International Journal of Business and Society 14(3), 428 - 443.

Grassi, S. and Santucci De Magistris, P. (2013). It's all about volatility (of volatility): evidence from a two-factor stochastic volatility model. CREATES Research Paper 2013-03.

Gupta, R.., Hammoudeh, S., Kim, W.J. and Simo-Kengne, B.D. (2014) Forecasting China's foreign exchange reserves using dynamic model averaging: The role of macroeconomic fundamentals, financial stress and economic uncertainty. North American Journal of Economics and Finance,28, 170-189 .

Ismail, Z., A., Yahya, A. and Shabri, A. (2009). Forecasting gold prices using multiple linear Regression method. American Journal of Applied Sciences 6(8): 1509-1514.

Jaffe, J. (1989). Gold and gold stocks as investments for institutional portfolios. Financial Analysts Journal 45, 53-59. 
Jensen, G. R., Johnson, R. R. and Mercer, J. M. (2002). Tactical asset allocation and commodity futures. The Journal of Portfolio Management 28(4), 100-111.

Koop, G. and D. Korobilis (2011). UK macroeconomic forecasting with many predictors: Which models forecast best and when do they do so? Economic Modelling 28, 2307-2318.

Koop, G. and D. Korobilis (2012). Forecasting inflation using dynamic model averaging. International Economic Review 53, 867-886.

Lili, L. and Chengmei, D. (2013). Research of the influence of macroeconomic factors on the price of gold. Procedia Computer Science 17, 737-743.

Pindyck, R. S. and Rotemberg, J. J. (1990). The excess co-movement of commodity prices. The Economic Journal 100, 1173-1189.

Pierdzioch, C., Risse, M. and Rohloff, S. (2014a). On the efficiency of the gold market: Results of a real-time forecasting approach. International Review of Financial Analysis 32, 95-108.

Pierdzioch, C., Risse, M. and Rohloff, S. (2014b). The International business cycle and gold-price fluctuations. The Quarterly Review of Economics and Finance (in press).

Raftery, A.E., Kárný, M. and P. Ettler (2010). Online prediction under model uncertainty via dynamic model averaging: application to a cold rolling mill. Technometrics 52(1), 52-66.

Shafiee, S. and Topal, E. (2009). When will fossil fuel reserves be diminished? Energy Policy 37 (1), 181-189.

Sjaastad. L. J. (2008). The price of gold and the exchange rates: Once again. Resources Policy 33, 118-124.

Sjaastad, L. J and Scacciavillani, F. (1997). The price of gold and the exchange rate. Journal of International Money and Finance 15 (6), 879-897.

Tortora, A. D. 2010. Exchange rate forecasting: Bayesian model averaging and structural instability. Available from

http://www.webmeets.com/files/papers/SAEE/2010/538/BMA_FX_SJM.pdf.

Wright, J. H. (2008). Bayesian model averaging and exchange rate forecasts. Journal of Econometrics 146, 329-341. 


\section{Data Appendix}

Table A1: Variables used in predicting the gold price returns

\begin{tabular}{|c|c|c|c|}
\hline Category & Tcode* & Description & Source \\
\hline \multirow{4}{*}{$\begin{array}{l}\text { Business Cycle } \\
\text { Factor } \\
\text { (BUSCYCLE) }\end{array}$} & 4 & EU Industrial Production & Global Insight \\
\hline & 4 & China Industrial Production & China National Bureau of Statistics \\
\hline & 4 & US Industrial Production & FRED \\
\hline & 4 & Japan Industrial Production & $\begin{array}{l}\text { Ministry Of Economy, Trade And } \\
\text { Industry }\end{array}$ \\
\hline \multirow{6}{*}{$\begin{array}{l}\text { Nominal Factor } \\
\text { (NORMFACTOR) }\end{array}$} & 4 & EU CPI & Global Insight \\
\hline & 4 & China CPI & China National Bureau of Statistics \\
\hline & 4 & US CPI & Bureau of Labor Statistics \\
\hline & 4 & Japan CPI & Statistics Bureau \\
\hline & 4 & EU M1 Index & Global Insight \\
\hline & 4 & US M1 (Bill. USD) & FRED \\
\hline \multirow{3}{*}{$\begin{array}{l}\text { Interest Rate } \\
\text { Factor } \\
\text { (INTEREST) }\end{array}$} & 4 & EU Policy Rate & Global Insight \\
\hline & 4 & 1 Month LIBOR on British Pounds & British Bankers' Association \\
\hline & 4 & US Policy Rate & FRED \\
\hline \multirow{6}{*}{$\begin{array}{l}\text { Commodity Price } \\
\text { Factor } \\
\text { (COMMPRICE) }\end{array}$} & 4 & $\begin{array}{l}\text { CRB US Spot Commodity Price } \\
\text { Index }\end{array}$ & Datastream \\
\hline & 4 & All Commodity Price Index & $\mathrm{IMF}$ \\
\hline & 4 & $\begin{array}{l}\text { Dated Brent, light blend } 38 \text { API, fob } \\
\text { U.K. }\end{array}$ & IMF \\
\hline & 4 & $\begin{array}{l}\text { Dubai, medium, Fateh } 32 \text { API, fob } \\
\text { Dubai Crude Oil }\end{array}$ & $\mathrm{IMF}$ \\
\hline & 4 & $\begin{array}{l}\text { West Texas Intermediate } 40 \text { API, } \\
\text { Midland Texas }\end{array}$ & IMF \\
\hline & 4 & $\begin{array}{l}\text { Silver Fixing Price (US cents per troy } \\
\text { ounce) }\end{array}$ & $\begin{array}{l}\text { London Bullion Market } \\
\text { Association }\end{array}$ \\
\hline \multirow{3}{*}{$\begin{array}{l}\text { Exchange Rate } \\
\text { Factor } \\
(\text { EXCH) }\end{array}$} & 4 & $\begin{array}{l}\text { US effective exchange rate } \\
\text { (foreign/dollar) }\end{array}$ & FRED \\
\hline & 4 & Yen/dollar & FRED \\
\hline & 4 & dollar/euro & Global Insight \\
\hline \multirow{4}{*}{$\begin{array}{l}\text { Stock Market } \\
\text { Factor } \\
(\text { STOCK) }\end{array}$} & 4 & $\begin{array}{l}\text { The STOXX Europe } 600 \text { Index } \\
\text { represents large, mid and small } \\
\text { capitalization companies across } 18 \\
\text { countries of the European region. }\end{array}$ & STOXX (www.stoxx.com) \\
\hline & 4 & $\begin{array}{l}\text { The STOXX North America } 600 \\
\text { Index represents the largest } \\
\text { companies in the Americas region. }\end{array}$ & STOXX (www.stoxx.com) \\
\hline & 4 & $\begin{array}{l}\text { The STOXX Asia/Pacific } 600 \text { Index } \\
\text { represents the largest companies in } \\
\text { Australia, Hong Kong, Japan, New } \\
\text { Zealand and Singapore. }\end{array}$ & STOXX (www.stoxx.com) \\
\hline & 4 & MSCI_emerging & MSCI (www.msci.com) \\
\hline KCFSI & 1 & $\begin{array}{l}\text { The Kansas City Fed's Financial } \\
\text { Stress Index }\end{array}$ & Kansas City Fed \\
\hline EPU_US & 1 & $\begin{array}{l}\text { Economic Policy Uncertainty Index } \\
\text { for United States }\end{array}$ & $\begin{array}{l}\text { Scott Baker, Nicholas Bloom and } \\
\text { Steven J. Davis }\end{array}$ \\
\hline
\end{tabular}

Note: 1 and 4 in Tcode (transformation code) indicate no transformation (level) and the first-difference of the logarithm of the variable, respectively. 